\title{
Informação e conhecimento na inovação e no desenvolvimento local
}

\author{
Sarita Albagli \\ Doutorado em geografia. \\ Universidade Federal do Rio de Janeiro, UFRJ, Rio de Janeiro. \\ E-mail:sarita@ibict.br \\ Maria Lucia Maciel \\ Socióloga pela Universidade de Brasília. \\ Doutora em sociologia pela Université de Paris. \\ E-mail:mlmaciel@centroin.com.br
}

INTRODUÇÃO

Nas transformações em curso desde as últimas décadas do século XX, projeta-se o papel estratégico da informação e do conhecimento em diferentes dimensões da vida em sociedade. Um aspecto que é hoje objeto de crescente atenção nesse debate diz respeito à indissociabilidade entre as dinâmicas cognitiva, informacional, inovativa e socioespacial.

Este artigo parte do reconhecimento de que a produção, a socialização e o uso de conhecimentos e informações, assim como a conversão destes em inovações, constituem processos socioculturais e que tais práticas e relações inscrevem-se no espaço e na própria produção do espaço, em suas várias escalas.

Boa parte da literatura mais recente sublinha especificamente a importância do conhecimento tácito como fonte de inovação e competitividade, bem como o papel das interações locais na produção e na difusão desse conhecimento (Lundvall, 2002; Patrucco, 2003; Albagli; Maciel, 2003). Daí a conexão entre esse debate e noções como as de capital social, territorialidade, redes, bem como das chamadas aglomerações produtivas - distritos industriais, clusters, milieux innovateurs, arranjos e sistemas produtivos e inovativos locais.

Há, no entanto, lacunas no sentido de se definirem e desenvolverem metodologias e instrumentos de pesquisa que demonstrem empiricamente a relevância dos fluxos locais de conhecimento para a inovação e que evidenciem os fatores socioespaciais que interferem nesses fluxos.

Este artigo procura primeiramente evidenciar as dimensões espaciais e socioinstitucionais da produção e difusão de conhecimentos e inovações, com base na revisão e síntese interpretativa da literatura. Em seguida, indicam-se algumas pistas para o desenvolvimento de parâmetros e instrumentos de pesquisa empírica que permitam incorporar tais dimensões na análise da dinâmica inovativa local, particularmente no que tange à produção e ao compartilhamento de informações e conhecimentos. Nas considerações finais, sugere-se uma agenda de pesquisa que permita prosseguir no aprofundamento e elucidação das questões tratadas no trabalho. 


\section{DIMENSÃO SOCIOESPACIAL}

A importância da informação e do conhecimento no mundo contemporâneo tem sido usualmente associada ao desenvolvimento das tecnologias de informação e comunicação (TICs), que, nas últimas décadas, transformaram as formas de produzir e distribuir bens materiais e imateriais, assim como as percepções de espaço e de tempo. Daí que, em diferentes abordagens sobre o que melhor caracteriza e distingue o cenário atual, há claramente ênfase tanto nessa dimensão tecnológica, quanto nos aspectos econômicos que lhes estão subjacentes.

Mas, contrariamente à noção de que as novas TICs representam o declínio da importância da dimensão territorial, as atuais mudanças técnico-econômicas, ditas "globalizantes", têm sido acompanhadas da valorização das diferenças socioespaciais. Ante as novas tecnologias, que alimentam os processos de globalização, o local redefinese, ganhando densidade "comunicacional" e técnica, tanto como nó das redes de comunicação global, quanto pela sua dinâmica interna.

Ampliação das redes telemáticas demonstrou também não ser suficiente para incorporar os diversos países, regiões e segmentos sociais ao novo padrão. As TICs fornecem a base técnica para os novos modos de reprodução e valorização do capital - seja o capital financeiro, transformado em pura informação, seja o capital produtivo -, ao permitirem a flexibilização do aparato técnico e do trabalho e ao viabilizarem a produção e a circulação de um conjunto de bens informacionais de ágil produção, comercialização e consumo. Não necessariamente, porém, promovem maior socialização de conhecimentos estratégicos, nem evitam o crescente agravamento das desigualdades sociais e territoriais.

Neste contexto, verifica-se, no debate atual sobre esses temas, uma relativa convergência em torno dos seguintes pontos: (a) a distinção entre os conceitos de informação e de conhecimento e o acesso aos mesmos (Lundvall, 2002); (b) a maior importância relativa do chamado conhecimento tácito diante do conhecimento codificado, distinção esta introduzida por Michael Polanyi, na década de 1950, ao assinalar que o que sabemos é mais do que conseguimos dizer ou descrever, sendo recentemente popularizada por Nonaka e Takeuhi (1997); (c) o reconhecimento de que as novas TICs, ainda que proporcionando maior difusão de informações e conhecimentos codificados, não impedem a concentração espacial e social dos mesmos (Albagli, 1997).

Reconhece-se, do mesmo modo, que o conhecimento é socialmente moldado, possuindo não apenas uma dimensão temporal/histórica, mas também espacial/ territorial. Ainda que se possa fazer referência a um conceito genérico de conhecimento, os conhecimentos são específicos e diferenciados. Em um mesmo contexto econômico e sociocultural, o conhecimento diferenciase, segundo áreas e comunidades de especialistas; segmentos e agentes econômicos; segmentos e grupos sociais; empresas e organizações; constelações regionais e redes sociais e produtivas (Foray, 2000; Albagli e Maciel, 2003).

O conhecimento tácito, em particular, geralmente encontrase associado a contextos organizacionais e territoriais específicos, sendo transmitido e desenvolvido por meio de interações locais (Polanyi, 1966). E, considerando a maior facilidade de disseminação de conhecimentos codificados por meio das TICs, o conhecimento tácito é considerado diferencial básico de competitividade, assim como uma das principais fontes de inovação.

A circulação do conhecimento entre contextos diferenciados, passando de tácito a codificado e viceversa, envolve processos de "desterritorialização", quando é descontextualizado, e de "reterritorialização" (ou "recontextualização"), "que inclui o processo de aprendizado e sedimentação, quando o conhecimento se enraíza no território" (Yoguel, 1998, p.4).

A capacidade de gerar, de adaptar/recontextualizar e de aplicar conhecimentos, de acordo com as necessidades e especificidades de cada organização, país e localidade, é, portanto, central. Desse modo, tão importante quanto a capacidade de produzir novo conhecimento é a capacidade de processar e recriar conhecimento, por meio de processos de aprendizado; e, mais ainda, a capacidade de converter esse conhecimento em ação, ou, mais especificamente, em inovação. Isso é particularmente relevante no caso de países em desenvolvimento. Importa sobretudo compreender e conhecer "os mecanismos endógenos de criação de 'competências' e de transformação de conhecimentos genéricos em específicos" (Yoguel, 1998:4).

O aprendizado, por sua vez, não se limita a ter acesso a informações; consiste na aquisição e construção de diferentes tipos de conhecimentos, competências e habilidades. A informação serve fundamentalmente à circulação ou transporte de conhecimentos (Latour, 1987), mas não necessariamente gera conhecimento; não é, por si só, capaz de alterar estruturas cognitivas. O aprendizado deve ser pensado como relação social, como um processo em que "as pessoas não só são participantes ativos na prática de uma comunidade, mas também desenvolvem suas próprias identidades em relação àquela comunidade" (Hildreth e Kimble, 2002, p. 23). 
Esse debate reflete também a afirmação de um conceito mais abrangente de inovação para além da inovação tecnológica. Nesse, valoriza-se não apenas o conhecimento formalizado e dito avançado (conhecimento científicotecnológico), mas também o conhecimento nãoformalizado, construído nas práticas econômicas e socioculturais (Lall e Ghosh, 2002) - os conhecimentos de indivíduos, em seus papéis de trabalhadores, consumidores e cidadãos, de organizações públicas e privadas, de populações, comunidades e povos tradicionais, entre outros grupos e segmentos. Em contrapartida, boa parte do próprio conhecimento científico também é tácita, assim como o dito conhecimento prático pode ser, em parte, codificado.

Cada local ou região dispõe assim de diferentes combinações de características e bens coletivos - físicos, sociais, econômicos, culturais, políticos, institucionais - que influenciam sua capacidade de produzir conhecimento, de aprender e de inovar. E, no sistema de relações que configuram o ambiente local, a dimensão cognitiva dos atores - expressa em sua capacidade de tomar decisões estratégicas e em seu potencial de aprendizado e inovação - é determinante de sua capacidade de capitanear os processos de crescimento e mudança, ou seja, de desenvolvimento local (Barquero, 1999).

Nessas interações locais, desenvolve-se um conhecimento coletivo, o qual é diferenciado e desigualmente distribuído, podendo ou não constituir importante fonte de dinamismo para aquele ambiente. Esse conhecimento coletivo não corresponde simplesmente à soma de conhecimentos de indivíduos e organizações; resulta das sinergias geradas a partir dos vários tipos de interação; e altera-se inclusive na sua interseção com a circulação globalizada de informação e conhecimento.

As chamadas aglomerações produtivas, científicas, tecnológicas e/ou inovativas - tais como distritos industriais, clusters, milieux inovadores, arranjos produtivos locais, entre outros (Cassiolato e Lastres, 1999) - são consideradas ambientes propícios a interações, à troca de conhecimentos e ao aprendizado, por meios diversos, tais como a mobilidade local de trabalhadores; redes formais e informais; existência de uma base social e cultural comum que dá o sentido de identidade e de 'pertencimento'.

A localização ou proximidade espacial facilita maior interação e comunicação, mas não é, por si só, um fator determinante para tal - são necessárias também condições institucionais e socioculturais que as favoreçam.

\section{DIMENSÃO SOCIOINSTITUCIONAL}

As características sociais e políticas locais constituem então aspecto central para a ampliação da capacidade inovadora.

A difusão e o compartilhamento de informações e conhecimentos requerem que os atores estejam conectados, que haja canais ou mecanismos de comunicação que propiciem os vários fluxos de conhecimento e o aprendizado interativo.

Estudos mostram evidências da existência de uma correlação entre a presença de relações cooperativas, a diversidade de mecanismos de comunicação entre agentes diferenciados e o desempenho inovador das empresas (Patrucco, 2003). Demonstra-se que (1) organizações e agentes que cooperam introduzem maior número de inovações do que os que não cooperam e que (2) o grau de inovação aumenta com a variedade de parceiros comunicando-se e cooperando em rede.

A pluralidade de agentes contribui para que sejam geradas essas várias oportunidades de comunicação. Do mesmo modo, a pluralidade de distintas, mas complementares, relações cooperativas - mais do que a concentração em um tipo dominante de interação - é uma das principais fontes de inovação.

Supõem-se, ainda, um contexto social de comunicação e a existência de códigos compartilhados e reconhecidos pelos sujeitos da comunicação. Estes se inserem em condições explícitas (envolvendo símbolos e sinais) e tácitas (sua trajetória individual, o contexto cultural), seu capital simbólico e cultural (Bourdieu, 1989) suas competências lingüísticas (capacidade de compreender os termos da linguagem) e enciclopédicas (conhecimento em relação ao conteúdo da mensagem) (Sfez, 1996). Conforme assinalado por Sfez (1996:5),

o saber sobre o qual a comunicação das informações vai incidir já existe e serve para interpretá-la. Mas este saber é, naturalmente, formado por mensagens anteriores, geradas por uma aprendizagem social e vindas de uma herança cultural, irrigada pelas experiências pessoais.

Ganha importância assim compreender e promover as condições que propiciem a configuração de um sistema de comunicação múltiplo, favorecendo a interação e a cooperação local, bem como a difusão e o intercâmbio de diferentes tipos de informações, conhecimentos e inovações.

Nesse sentido, projetam-se as noções de territorialidade e de capital social. 
A noção de territorialidade procura evidenciar as interfaces entre as dimensões territorial e sociocultural. Territorialidade refere-se às relações entre um indivíduo ou grupo social e seu meio de referência, manifestandose nas várias escalas geográficas - uma localidade, uma região ou um país - e expressando um sentimento de pertencimento e um modo de agir em um dado território. A territorialidade reflete o vivido territorial em toda sua abrangência e em suas múltiplas dimensões - cultural, política, econômica e social. Ela se desenvolve a partir da coexistência dos atores sociais em um dado espaço geográfico, engendrando um sentimento de sobrevivência do coletivo e referências socioculturais comuns, ainda que considerada a diversidade de interesses ali presentes. A territorialidade, como atributo humano, é primariamente condicionada por valores e normas sociais, que variam de sociedade para sociedade, de um período para outro. A territorialidade não traduz, portanto, apenas uma relação com o meio; ela é uma relação triangular entre os atores sociais mediada pelo espaço (Albagli, 2004).

O conceito de capital social, que se difunde particularmente desde a década de 1990, expressa o reconhecimento e a valorização de recursos embutidos em estruturas sociais, até então não contabilizados por outras formas de capital. Contrariamente à visão da economia neoclássica, que supõe uma racionalidade estritamente econômica e individual, entende-se, dessa perspectiva, que os atores econômicos não são átomos isolados, mas encontram-se imersos (embedded) ${ }^{*}$ em relações e estruturas sociais.

O conceito de capital social surge no âmbito da sociologia. Sua origem remonta aos trabalhos de Pierre Bourdieu (1980), James Coleman (1988) e Robert Putnam (1993), sendo compreendido como o conjunto de instituições formais e informais, normas sociais, hábitos e costumes que afetam os níveis de confiança, solidariedade e cooperação em um grupo ou sistema social (AlbaglieMaciel, 2003). Na literatura sobre o tema, predomina atualmente a versão anglo-saxônica, que percebe capital social como sinônimo de consenso normativo, favorecendo o espírito cívico expresso em uma rica vida associativa, enfatizando

\footnotetext{
" A idéia de embeddedness foi primeiramente desenvolvida por Karl Polanyi, em 1944, em sua obra The Great Transformation, da seguinte forma: "The human economy [...] is embedded and enmeshed in institutions, economic and noneconomic. [...] religion or government may be as important to the structure and functioning of the economy as monetary institutions or the availability of tools and machines themselves that lighten the toil of labor" [citação de Polanyi, Aresberg and Pearson (1957), (Smelser e Swedberg, 1994)]. Posteriormente Mark Granovetter, na linha da "sociologia econômica", usou o conceito para argumentar que a ação econômica está incrustada (embedded) na estrutura social e na cultura. Ver: Granovetter (1973). "The strength of weak ties". American Journal of Sociology, 78/4, 1350-80; Granovetter (1985). "Economic action and social structure: the problem of embeddedness". American Journal of Sociology, 91, 3 (November):481-51
}

portanto as funções do capital social como fator de coesão da sociedade e de dinamismo econômico. A sociologia francesa, por sua vez, destaca, nessa discussão, as relações de poder, conflito e desigualdade inerentes às estruturas sociais, argumentando que o capital social, como todas as outras formas de capital, reflete essas desigualdades.

Entende-se que o capital social propicia (Lin et alii, 2001):

- maior facilidade de compartilhamento de informações e conhecimentos, bem como custos mais baixos, devido a relações de confiança, espírito cooperativo, referências socioculturais e objetivos comuns;

- melhor coordenação de ações e maior estabilidade organizacional, devido a processos de tomada de decisão coletivos;

- maior conhecimento mútuo, ampliando a previsibilidade sobre o comportamento dos agentes, reduzindo a possibilidade de comportamentos oportunistas e propiciando maior compromisso em relação ao grupo.

Se concebido como processo dinâmico de relações sociais em redes nas quais se constrói o conhecimento tácito, o capital social está intimamente ligado ao aprendizado interativo e à cooperação, podendo ainda facilitar as ações coletivas geradoras de arranjos produtivos articulados. Os recursos imateriais ou intangíveis, presentes nas redes sociais, quando direcionados para um esforço conjunto de desenvolvimento, convergem no que Hubert Schmitz (2000) chamou de "eficiência coletiva", envolvendo um complexo de interações sociais locais, que propiciam a produção e reprodução de conhecimento tácito, catalisando processos de inovação e difusão.

\section{PARÂMETROS DE PESQUISA EMPÍRICA}

O entendimento dessas complexas relações coloca novas questões nos campos conceitual e metodológico, demandando instrumentos capazes de verificar e avaliar os processos e a intensidade da circulação de informações e conhecimentos, bem como seu papel para o dinamismo socioeconômico local.

Nos últimos anos, a literatura especializada** vem apontando o alcance limitado de indicadores de conhecimento e de inovação tradicionalmente utilizados, tais como gastos em pesquisa e desenvolvimento, número

\footnotetext{
*** Ver, por exemplo, Foray (2000), Lall e Ghosh (2002), Lundvall (2002), OCDE (2001).
} 
de patentes e de inovações e dados relativos à educação formal (diplomas e certificados). Alguns consideram inclusive que a escolha desses indicadores, e não de outros, resulta menos de uma reflexão metodológica sobre sua pertinência e mais da maior disponibilidade de certos dados e informações (Yoguel,1998). Tais unidades de medida, embora fundamentais, não são consideradas hoje suficientes para uma avaliação dos esforços e resultados dos processos de aprendizado e de geração e uso de conhecimentos, já que refletem basicamente os aspectos formais da inovação e do aprendizado. A produção de qualquer produto ou serviço pode resultar em aprendizado e gerar conhecimento, mesmo que esse não tivesse sido o objetivo inicial. A dificuldade reside justamente em captar e avaliar os fluxos de conhecimento tácito, especialmente aqueles gerados de maneira não intencional.

Outro aspecto não adequadamente contemplado pelos indicadores tradicionais refere-se ao fluxo de conhecimentos, crucial quando se parte do princípio de que a construção do conhecimento é um processo social.

Por outro lado, a existência de um ambiente ou de um espaço público propício à interação e à difusão de informações e conhecimentos não implica automaticamente sua plena incorporação pelo conjunto dos agentes que ali se situam. É preciso examinar a capacidade e a competência interna de cada indivíduo e organização interagir e capitalizar os benefícios dessa interação, ou seja, sua capacidade de viabilizar a interlocução com outros indivíduos e organizações e de aprender com essa interlocução.

Identificam-se dois grandes tipos de estudos empíricos sobre processos de interação e cooperação (Foray, 2000). Um conjunto privilegia estudos de casos, apresentando descrições detalhadas sobre esses processos. Essa abordagem resiste a generalizações, bem como à codificação e à estratificação de informações em descritores, argumentando que a codificação estatística mutila, corta e reduz, perdendo a visão de conjunto. Outro tipo de estudo é fundamentalmente estatístico e supõe a possibilidade de generalizar, ou inferir, com determinado grau de confiabilidade, conhecimento aplicável ao todo.

O desafio que nos colocamos é, portanto, definir a natureza das relações locais que produzem resultados socioeconômicos propícios ao desenvolvimento baseado no conhecimento, no aprendizado e na inovação, procurando construir novos instrumentos de pesquisa empírica capazes de captar essas relações, seus canais e seus fluxos. Tais instrumentos tornam-se ainda mais relevantes à medida que subsidiem a formulação de políticas e estratégias mais adequadas a contextos específicos.

Como variáveis e parâmetros de análise, em estudos empíricos sobre a dimensão socioespacial do conhecimento, inovação e aprendizado em âmbito local, sugere-se o seguinte: a identificação e a caracterização dos atores-chave; o mapeamento dos tipos, formas e características das interações entre esses atores; a verificação do papel da proximidade territorial, do ponto de vista das práticas produtivas, da ação cooperativa e das fontes de informação e de conhecimento para a inovação; as interfaces entre o arcabouço institucional, os níveis de capital social e a dinâmica cognitiva e inovativa local; os canais, mecanismos e intensidade dos fluxos de conhecimento nas interações locais.

A primeira questão é identificar e caracterizar os atoreschave. A cooperação intencional, particularmente entre empresas, é a que tem predominado como objeto de estudos. Mas a inovação local não envolve apenas empresas e instituições de ensino e pesquisa, e sim uma pluralidade de outros atores que também detêm diferentes tipos de conhecimentos e competências relevantes aos sistemas produtivo e tecnológico.

De modo esquemático, as interações locais podem envolver os seguintes atores: (a) agentes econômicos (clientes, parceiros e competidores; fornecedores de insumos, componentes, ou equipamentos; fornecedores de serviços técnicos; matriz ou filial); (b) agentes de conhecimento (consultores; universidades e institutos de pesquisa); (c) agentes de regulação (governos em seus vários níveis); (e) demais atores sociais (sindicatos, associações empresariais organizações de suporte, organizações do chamado "Terceiro Setor", entre outros). Os atores podem ser também caracterizados como interno/externo ao contexto socioeconômico local; público/privado; conforme sua 'missão' ou finalidade; pela sua nacionalidade; entre outras características.

Identificados os atores-chave, trata-se de mapear e caracterizar as diversas formas de interação dos atores locais e desses com atores externos. Tais interações podem expressar-se tanto em relações de competição e conflito, quanto em relações de confiança e parceria, em níveis diferenciados. De modo tipificado, tem-se:

- ação ou influência recíproca, freqüentemente nãointencional, ocasionada a partir de contatos e relações com finalidades diversas e em situações diferenciadas (interação de vários tipos, em sentido amplo); 
- ação e/ou entendimento conjunto, para fins de interesse comum, por meios mais ou menos formais (articulação, cooperação, parceria);

- troca/permuta/transação de caráter comercial ou não (intercâmbio de informações e idéias; apoio técnico; venda/aquisição de serviços e produtos).

A caracterização do tipo de interação requer ainda informações sobre o número e os tipos de atores envolvidos; motivações e objetivos; procedimentos e mecanismos; freqüência, intensidade e duração; problemas e dificuldades das interações.

$\mathrm{Na}$ escolha do parceiro ou interlocutor, influem aspectos como confiança, reputação, qualificação (conhecimento técnico), proximidade, identidade e estabilidade institucional ou financeira. Os problemas e obstáculos à interação e, particularmente, à cooperação remetem, em grande parte, à dimensão institucional (em sentido amplo) e ao papel do capital social.

É possível então diferenciar e caracterizar ambientes mais propícios à cooperação - em que se verificam interações regulares, numericamente expressivas e de maior complexidade, além de relações formais e informais de cooperação; e ambientes onde os vínculos entre os agentes são escassos e descontínuos, com poucas relações formais e informais de cooperação e atividades conjuntas.

O ambiente institucional como um todo, no qual as empresas interagem, repercute na capacidade de inovação, a partir de uma "capacidade social" difusa, histórica e culturalmente moldada, de gerar e de apropriar-se de novos conhecimentos, bem como de utilizá-los em favor do desenvolvimento local.

\section{À GUISA DE CONCLUSÃO}

A chamada era da informação e do conhecimento, embora assumindo uma dimensão global, expressa: (a) a diferenciação entre realidades culturais e projetos de sociedade, ou seja, entre comunidades territoriais e segmentos sociais diversos; (b) a desigualdade entre sociedades com distintas condições de desenvolvimento, bem como entre segmentos de diferentes níveis socioeconômicos no interior de uma mesma sociedade.

A necessidade de melhor compreender essa diferenciação e essa desigualdade, seus fatores, possíveis desdobramentos e perspectivas tem motivado a atenção à dimensão socioespacial, para além da dimensão técnico-econômica stricto sensu. Trata-se de compreender informação, conhecimento e inovação como constructos socioculturais e como constituintes e expressões da dinâmica políticoinstitucional, sendo, portanto, moldados no tempo/história e no espaço/território.

Nesse sentido, noções como as de capital social e de territorialidade ganham espaço na reflexão sobre esses temas nas várias áreas do conhecimento, contribuindo para melhor desvendar as diferenças entre os processos de inovação e seus resultados em situações específicas. No entanto, verifica-se que tais noções são vistas, ainda, como "externalidades": como contexto ou complemento na análise.

Em síntese, na perspectiva por nós adotada, não existe um espaço informacional, cognitivo e inovativo autônomo de um espaço social e institucional, do mesmo modo que tais espaços adquirem e conferem contornos específicos em cenários territoriais concretos.

Tal suposto deve também refletir-se nos instrumentos de pesquisa empírica sobre os processos de inovação e aprendizado de base territorial, tal como se observa nos estudos sobre aglomerações produtivas, em suas várias modalidades.

Propomos então uma abordagem metodológica que situe a análise nessa dimensão socioespacial. Trata-se não apenas de identificar, distinguir e definir o ambiente institucional e cultural específico em questão, composto de suas especificidades políticas, sociais e econômicas. Mais que isso, é preciso desenvolver parâmetros e instrumentos teórico-metodológicos que permitam incorporar tal dimensão na própria pesquisa sobre a intensidade, as condições, os obstáculos e os resultados da geração e difusão de informações e conhecimentos, no âmbito das diversas formas de interação no território.

Sugere-se assim a relevância de ampliar o escopo e a abrangência de análise para além dos indivíduos e organizações tomados individualmente, para considerar centralmente suas interações, focando nos condicionantes e resultados dos fluxos de conhecimentos entre os atores locais e destes com atores externos e, particularmente, verificando e avaliando o papel da proximidade territorial e da dinâmica socioinstitucional. Aglomerações produtivas constituem cenário territorial propício à análise, já que são potencialmente espaços privilegiados de conhecimento, aprendizado e inovação interativos. Não devem ser, no entanto, compreendidas apenas como espaços econômicos, mas sobretudo como ambientes sociais em toda sua complexidade.

Desse ponto de vista, a compreensão da dinâmica cognitiva e de inovação local deve focar a análise dos seguintes aspectos: 
a) os processos de geração, difusão e uso de conhecimentos, especialmente aqueles derivados das particularidades da cultura produtiva local, bem como os conhecimentos sobre com quem cooperar e interagir (know who);

b) o conhecimento e o aprendizado resultantes das interações locais, particularmente aqueles gerados de modo não-intencional;

c) não apenas a cooperação formalmente estabelecida, mas também e principalmente os vários tipos de interação informal;

d) o caráter sistêmico do aprendizado e da inovação, reconhecendo o papel de cada ator local para a geração do conhecimento coletivo e de uma inteligência local;

e) os canais de comunicação entre os agentes, como parte essencial do sistema de inovação local, e a diversidade institucional como fator crucial das oportunidades de comunicação;

f) os resultados não apenas para a competitividade dos agentes econômicos, mas também e sobretudo para o desenvolvimento socioeconômico local;

g) a capacidade de cada organização interagir e cooperar, bem como, a partir dessas relações, gerar conhecimento e promover o aprendizado e a inovação.

A avaliação do papel do capital social e da territorialidade, como fatores de cooperação, de compartilhamento de conhecimentos e experiências e de aprofundamento de vínculos entre os atores locais, constitui um dos principais desafios dessa análise.

Finalmente, cabe assinalar que esse debate no campo teórico-metodológico implica importantes desdobramentos na proposição de políticas, particularmente as de cunho territorial, ante o reconhecimento da centralidade da dimensão cognitiva dos processos e estratégias de desenvolvimento local/regional. Isso porque cada território é continente de conhecimento específico e estratégico, e a sua desestruturação tem por conseqüência também a "desconstrução" do conhecimento associado.

Traçar tais políticas requer o conhecimento das condições locais específicas, suas carências e potencialidades, conhecimento que pode ser obtido em pesquisas empíricas tais como as sugeridas. Coloca-se então a centralidade de reconhecer e capitalizar os conhecimentos específicos de cada território. $\mathrm{O}$ conhecimento gestado a partir da realidade e das necessidades locais é relevante tanto para se obter vantagem competitiva, transformando as características e atributos específicos de cada território em valorização econômica, como também para promover padrões de desenvolvimento mais sustentáveis, em termos sociopolíticos, econômicos e ambientais.

Artigo recebido em 25-10-2004 e aceito para publicação em 29-03-2005.

\section{Agradecimentos}

Agradecemos o apoio do CNPq e da FARJ.

\section{REFERÊNCIAS}

ALBAGLI, Sarita. Informação e geopolítica contemporânea: o papel dos sistemas de propriedade intelectual. Informare, v. 3, n. 1/2, 1997.

. Globalização e espacialidade: o novo papel do local. In: CASSIOLATO, José Eduardo; LASTRES, Helena M. M. Globalização e inovação localizada: experiências de sistemas locais no Mercosul. Brasília : IBICT/IEL, 1999.

Território e territorialidade. In: LAGES, Vinícius; BRAGA, Christiano; MORELLI, Gustavo. Territórios em movimento: cultura e identidade como estratégia de inserção competitiva. Rio de Janeiro : Relume Dumará; Brasília : Sebrae, 2004.

; MACIEL, Maria Lúcia. Capital social e desenvolvimento local In: LASTRES, Helena M. M.; CASSIOLATO, José Eduardo; MACIEL, Maria Lucia (Org.). Pequena empresa: cooperação e desenvolvimento local. Rio de Janeiro : Relume Dumará, 2003. p. 423-440.

BARQUERO, Antonio Vázquez. Desarrollo, redes e innovación. Madrid : Pirámide, 1999.

BOURDIEU, Pierre. Le capital social: notes provisoires. Actes de la Recherche en Sciences Sociales, n. 31, janv. 1980.

. Langage et pouvoir simbolique. Paris : Seuil, 1989.

BROWN, Thomas. Theoretical perspectives on social capital. Disponível em: $<$ http://hal.lamar.edu/ BROWNTF/SOCCAP.HTML>.

CASSIOLATO, José Eduardo; LASTRES, Helena M. M. Globalização e inovação localizada: experiências de sistemas locais no Mercosul. Brasília : IBICT/IEL, 1999.

COLEMAN, James S. Social capital in the creation of human capital. American Journal of Sociology, n. 94, p. 95-121, 1988. Supplement.

ERICKSON, Christopher L.; JACOBY, Sanford M. The effect of employer networks on workplace innovation and training. [S. 1.] : Industrial e Labor Relations, 2002.

FORAY, Dominique. Characterising the knowledge base: available and missing indicators. In: ORGANIZAÇÃO PARA A COOPERAÇÃO E O DESENVOLVIMENTO ECONÔMICO. Knowledge management in the learning society. Paris, 2000.

GIDDENS, Anthony. As conseqüências da modernidade. São Paulo : Unesp, 1991.

GRANOVETTER, Mark. Economic action and social structure: the problem of embeddedness. American Journal of Sociology, v. 91, n. 3, p. 481, Nov. 1985. 


\section{Sarita Albagli / Maria Lucia Maciel}

HARVEY, D. A condição pós-moderna. São Paulo : Loyola, 1993. 349 p.

HILDRETH, P. J.; KIMBLE, C. The duality of knowledge. Information Research, v. 8, n. 1, 2002. Disponível em: <http://nformationR.net/ir/81/paper142.html>.

INGLEHART, Ronald. Modernization and postmodernization: cultural, economic and political change in 43 societies. Princeton : Princeton University, 1997.

KRISTENSEN, Preben S.; MADSEN, Poul T.; VINDING, Anker L. Methodology and data collection (1997-1998) in the disko survey on product development collaboration. Aalborg, Dinamarca : University of Aalborg, 1999.

LALL, Somik V.; GHOSH, Sudeshna. Learning by dining: informal networks and productivity in Mexican industry: [S. 1.] : World Bank Development Research Group, 2002. (Policy research working paper, n. 2789).

LASTRES, Helena M. M.; LEGEY, Liz-Rejane I.; ALBAGLI, Sarita. Indicadores da economia e sociedade da informação, conhecimento e aprendizado In: VIOTTI, Eduardo B.; MACEDO, Mariano M. (Ed.). Indicadores de ciência, tecnologia e inovação no Brasil. Campinas : Editora da Unicamp, 2003. p. 533-578.

LATOUR, Bruno. Science in action. Stratfor : Open University, 1987.

LAURSEN, Keld; CHRISTENSEN, Jesper Lindgaard. The creation, distribution and use of knowledge: a pilot study of the Danish innovation system. Aalborg, Dinamarca : University of Aalborg, 1996.

LIN, N.; COOK, K.; BURT, R. (Ed). Social capital: theory and research. New York : Aldine de Gruyter, 2001.

LOCKE, R. Building trust. In: ANNUAL MEETINGS OF THE AMERICAN POLITICAL SCIENCE ASSOCIATION, 2001. [S.I. : s. n., 2001?].

LUNDVALL, B. A. Innovation, growth and social cohesion: the Danish model. Cheltenham : Edward Elgar Publishing, 2002.

MACIEL, Maria Lucia. Ciência, tecnologia e inovação: a relação entre conhecimento e desenvolvimento. BIB, n. 54, 2003.

. Knowledge and development: alternative perspectives and strategies. In: POLITICAL AND ETHICAL KNOWLEDGE ON ECONOMIC ACTIVITIES, 1., 2002, Santiago. [S. l. : s. n. 2002?].

. O milagre italiano: caos, crise e criatividade. Rio de Janeiro : Relume Dumará; Brasília : Paralelo 15, 1996.

NARAYAN, D.; CASSIDY, M. A dimensional approach to measuring social capital: development and validation of a social capital inventory. Current Sociology, v. 49, n. 2, 2001.
NONAKA, I.; TAKEUCHI, H. Criação de conhecimento na empresa: como as empresas japonesas geram a dinâmica da inovação. Campus : Rio de Janeiro, 1997.

OCDE. New science and technology indicators: STI Review, Paris, 2001. Special issue.

PATRUCCO, Pier Paolo. Institutional variety, networking and knowledge exchange: communication and innovation in the case of the Brianza technological district. Regional Studies, v. 37, n. 14, p. 159, Apr. 2003.

POLANYI, Michael. The tacit dimension. London : Routledge e Kegan Paul, 1966.

PUTNAM, R. Making democracy work: civic traditions in modern Italy. Princeton : Princeton University,1993.

RAFFESTIN, Claude. Por uma geografia do poder. Rio de Janeiro : Zahar, 1993.

REDESIST. Questionário completo: projeto arranjos e sistemas produtivos locais e as novas políticas de desenvolvimento industrial e tecnológico. [S. 1.], 2002. Disponível em: < http://www.ie.ufrj.br/redesist $>$.

SELIGSON, Mitchell A; RENNÓ, Lúcio R. Mensurando confiança interpessoal: notas acerca de um conceito multidimensional. Dados, v. 43, n. 4, 2000.

SFEZ, Lucien Informação, saber e comunicação. Informare - Caderno do Programa de Pós-Graduação em Ciência da Informaç̧ão, Rio de Janeiro, v. 2, n. 1, p. 5-13, 1996.

SCHMITZ, Hubert. Clusters and chains: how inter-firm organisation influences industrial upgrading. In: CASSIOLATO, José Eduardo; LASTRES, Helena M. M.; MACIEL, Maria Lúcia (Org.). Systems of innovation and development: evidence from Brazil. Cheltenham, G.B : [s. n.], 2003.

SMELSER, Neil J.; SWEDBERG, Richard. The sociological perspective on the economy. In: SMELSER, Neil J.; SWEDBERG, Richard. (Ed.). Handbook of economic sociology. Princeton : Princeton University, 1994.

SOJA, Edward. Postmodern geographies. Londres : Verso, 1989.

WOOLCOCK, M. The place of social capital in understanding social and economic outcomes. In: INTERNATIONAL SYMPOSIUM ON THE

CONTRIBUTION OF HUMAN AND SOCIAL CAPITAL TO SUSTAINED ECONOMIC GROWTH AND WELL-BEING, 2000, Quebec. [S. 1.] : OECD/ Human Resources Development, [2000?].

YOGUEL, Gabriel. Desarollo del proceso de aprendizaje de las firmas: los espacios locales y las tramas productivas. In: SEMINÁRIO GLOBALIZAÇÃO E INOVAÇÃO LOCALIZADA, 1998, Mangaratiba, RJ. [S. 1. : s. n. 1998?]. 